\title{
AN EXTENSION OF THE MARCINKIEWICZ INTERPOLATION THEOREM TO LORENTZ SPACES ${ }^{1}$
}

BY RICHARD A. HUNT

Communicated by A. Zygmund, June 22, 1964

The purpose of this paper is three-fold. First, the theorem of Marcinkiewicz on interpolation of operators (see [9, pp. 111-116]) is generalized to Lorentz spaces [5]. Second, this result is shown to be a rather easy consequence of a celebrated inequality of Hardy [2, pp. 245-246]:

TheOREM (HARDY). If $q \geqq 1, r>0$ and $f \geqq 0$, then

$$
\begin{aligned}
& \left(\int_{0}^{\infty}\left(\int_{0}^{t} f(y) d y\right)^{q} t^{-r-1} d t\right)^{1 / q} \leqq \frac{q}{r}\left(\int_{0}^{\infty}(y f(y))^{q} y^{-r-1} d y\right)^{1 / q} ; \\
& \left(\int_{0}^{\infty}\left(\int_{t}^{\infty} f(y) d y\right)^{q} t^{r-1} d t\right)^{1 / q} \leqq \frac{q}{r}\left(\int_{0}^{\infty}(y f(y))^{q} y^{r-1} d y\right)^{1 / q} .
\end{aligned}
$$

Third, previously open questions concerning the Marcinkiewicz Theorem are settled by showing our result is best possible.

Consider complex-valued, measurable functions $f$ defined on a measure space $(M, m)$. The distribution function of $f$ is defined by

$$
\lambda(y)=\lambda_{f}(y)=m\{x \in M:|f(x)|>y\}, \quad y>0 .
$$

$\lambda(y)$ is nonincreasing and continuous from the right. The nonincreasing rearrangement of $f$ onto $(0, \infty)$ is then defined by

$$
f^{*}(t)=\inf \left\{y>0: \lambda_{f}(y) \leqq t\right\}, \quad t>0 .
$$

$f^{*}(t)$ is also continuous from the right and has the same distribution function as $f$. The Lorentz spaces $L(p, q)$ are defined to be the collection of all $f$ such that $\|f\|_{p q}^{*}<\infty$, where

(4) $\|f\|_{p q}^{*}= \begin{cases}\left(\int_{0}^{\infty}\left(t^{1 / p} f^{*}(t)\right)^{q} \frac{d t}{t}\right)^{1 / q}, & 0<p<\infty, 0<q<\infty, \\ \sup _{t>0} t^{1 / p} f^{*}(t), & 0<p \leqq \infty, q=\infty .\end{cases}$

It is not hard to show that

1 This research, which is part of a $\mathrm{Ph} . \mathrm{D}$. thesis under the direction of Professor Guido Weiss, was supported by the U. S. Army Contract DA-31-124-ARO(D)-58. 


$$
\|f\|_{p s}^{*} \leqq B_{p, q, s}\|f\|_{p q}^{*}, \quad 0<q \leqq s \leqq \infty ;
$$

thus $L(p, q) \subset L(p, s)$.

Note that $\|f\|_{p p}^{*}$ is the $L^{p}$-norm of $f, 1 \leqq p \leqq \infty$. However, in general, $\|f\|_{p q}^{*}$ is not a norm since the triangle inequality may fail. It is customary (see [6]) to define a norm for certain $L(p, q)$ in the following way: Let

$$
f^{* *}(t)=\frac{1}{t} \int_{0}^{t} f^{*}(y) d y, \quad y>0
$$

and

$$
\|f\|_{p q}= \begin{cases}\left(\int_{0}^{\infty}\left(t^{1 / p} f^{* *}(t)\right)^{q} \frac{d t}{t}\right)^{1 / q}, & 1<p<\infty, 1 \leqq q<\infty, \\ \sup _{t>0} t^{1 / p} f^{* *}(t), & 1<p<\infty, q=\infty .\end{cases}
$$

$\|f\|_{p q}$ is equivalent to $\|f\|_{p q}^{*}$ in the sense that

$$
\|f\|_{p q}^{*} \leqq\|f\|_{p q} \leqq\left(\frac{p}{p-1}\right)\|f\|_{p q}^{*}, \quad 1<p<\infty, 1 \leqq q \leqq \infty .
$$

These $L(p, q)$, with the norm $\|f\|_{p q}$, are then Banach spaces.

An operator $T$ acting on a function space and having values that are functions is called quasi-linear if $T(f+g)$ is uniquely defined whenever $T f$ and $T g$ are defined, and if

$$
|T(f+g)| \leqq K(|T f|+|T g|),
$$

where $K$ is a constant independent of $f$ and $g$.

(2), (3), and (9) imply that

$$
(T(f+g))^{*}(t) \leqq 2 K\left((T f)^{*}\left(\frac{t}{2}\right)+(T g)^{*}\left(\frac{t}{2}\right)\right) .
$$

Our main result is the following

TheOrem. If $T$ is quasi-linear and

$$
\|T f\|_{p^{\prime} i^{\prime} i}^{*} \leqq B_{i}\|f\|_{p_{i} q_{i}}^{*}, \quad i=0,1, p_{0}<p_{1}, p_{0}^{\prime} \neq p_{1}^{\prime},
$$

then

$$
\|T f\|_{p^{\prime} \theta^{s}}^{*} \leqq B_{\theta}\|f\|_{p_{\theta} q}^{*},
$$

where $q \leqq s$, for $0<\theta<1,1 / p_{\theta}=(1-\theta) / p_{0}+\theta / p_{1}, 1 / p_{\theta}^{\prime}=(1-\theta) / p_{0}^{\prime}$ $+\theta / p_{1}^{\prime}, B_{\theta}=O(1 / \theta)$ as $\theta \rightarrow 0$ and $B_{\theta}=O(1 /(1-\theta))$ as $\theta \rightarrow 1$. 
The most interesting case of the theorem occurs when $1 \leqq q \leqq \infty$. For simplicity we consider only this case. We then see, using (5), that the hypotheses (11) are weakest in the form

$$
\|T f\|_{p^{\prime} i \infty}^{*} \leqq B_{i}\|f\|_{p_{i} 1}^{*}, \quad i=0,1, p_{0}<p_{1}, p_{0}^{\prime} \neq p_{1}^{\prime},
$$

with $\|f\|_{p_{1} 1}^{*}$ replaced by $\|f\|_{\infty, \infty}^{*}$ if $p_{1}=\infty$.

Again using (5), it is not hard to see what modifications are necessary to establish the general case.

Special cases of our theorem have appeared in various places. Krein and Semenov [3] proved that for $1 \leqq p \leqq \infty,(13)$ implies (12) with $s=q=1$. They also proved that if $T$ is linear, (13) implies (12) with $s=q=\infty$, and, in addition, that (13) is equivalent to the restricted weak type hypotheses of Stein and Weiss (see [8]). Hence, Stein and Weiss have proved that (13) with $1 \leqq p_{i} \leqq p_{i}^{\prime} \leqq \infty, i=0,1$, imply (12) for $s=p_{\theta}^{\prime}, q=p_{\theta}$.

Calderón [1] has shown, for $T$ linear, that (13) implies (12) when $q<s$.

Again with $T$ linear, the theorem, restricted to the $L(p, q)$ that are Banach spaces, can be derived from the general interpolation theory of Lions and Peetre [4] together with Peetre's identification of Lorentz spaces as spaces of means (see [7]).

Proof of the theorem. (5) implies that it is sufficient to prove (12) with $s=q$. Let $p=p_{\theta}$ and $p^{\prime}=p_{\theta}^{\prime}$. Put

$$
\begin{aligned}
& f^{t}(x)= \begin{cases}f(x) & \text { if }|f(x)|>f^{*}\left(t^{\gamma}\right), \\
0 & \text { otherwise; }\end{cases} \\
& f_{t}(x)=f(x)-f^{t}(x),
\end{aligned}
$$

where

$$
\gamma=\frac{\frac{1}{p_{0}^{\prime}}-\frac{1}{p^{\prime}}}{\frac{1}{p_{0}}-\frac{1}{p}}=\frac{\frac{1}{p^{\prime}}-\frac{1}{p_{1}^{\prime}}}{\frac{1}{p}-\frac{1}{p_{1}}} .
$$

(2) and (3) imply that

$$
\begin{aligned}
& f^{*}(y) \leqq \begin{cases}f^{*}(y), & 0<y<t^{\gamma}, \\
0, & y \geqq t^{\gamma}\end{cases} \\
& f_{t}^{*}(y) \leqq \begin{cases}f^{*}\left(t^{\gamma}\right), & 0<y<t^{\gamma}, \\
f^{*}(y), & y \geqq t^{\gamma} .\end{cases}
\end{aligned}
$$


Case 1. $p_{1}<\infty$ and $q<\infty$.

Using (10), a change of variables and Minkowski's inequality, we have

$$
\begin{aligned}
&\|T f\|_{p^{\prime} q}^{*} \leqq(2 K) 2^{1 / p^{\prime}} {\left[\left(\int_{0}^{\infty}\left(t^{1 / p^{\prime}}\left(T f^{t}\right)^{*}(t)\right)^{q} \frac{d t}{t}\right)^{1 / q}\right.} \\
&\left.+\left(\int_{0}^{\infty}\left(t^{1 / p^{\prime}}\left(T f_{t}\right)^{*}(t)\right)^{q} \frac{d t}{t}\right)^{1 / q}\right]
\end{aligned}
$$

which, by (13), is majorized by

$$
\begin{aligned}
(2 K) 2^{1 / p^{\prime}} & {\left[\left(\int_{0}^{\infty}\left(B_{0} t^{1 / p^{\prime}-1 / p^{\prime} 0}\left\|f_{t}\right\|_{p_{0} 1}^{*}\right)^{q} \frac{d t}{t}\right)^{1 / q}\right.} \\
+ & \left.\left(\int_{0}^{\infty}\left(B_{1} t^{1 / p^{\prime}-1 / p^{\prime} 1}\left\|f_{t}\right\|_{p_{1} 1}^{*}\right)^{q} \frac{d t}{t}\right)^{1 / q}\right] .
\end{aligned}
$$

Using (15) and Minkowski's inequality again, we can dominate this last sum by

$$
\begin{aligned}
(2 K) 2^{1 / p^{\prime}}\left[B_{0}\left(\int_{0}^{\infty}\left(t^{-\left(1 / p^{\prime}-1 / p^{\prime}\right)} \int_{0}^{t^{\gamma}} f^{*}(y) y^{1 / p_{0}-1} d y\right)^{q} \frac{d t}{t}\right)^{1 / q}\right. \\
+B_{1}\left(\int_{0}^{\infty}\left(t^{\left(1 / p^{\prime}-1 / p^{\prime}\right)} \int_{t^{\gamma}}^{\infty} f^{*}(y) y^{1 / p_{1}-1} d y\right)^{q} \frac{d t}{t}\right)^{1 / q} \\
\left.+B_{1}\left(\int_{0}^{\infty}\left(t^{\left(1 / p^{\prime}-1 / p^{\prime}\right)} \int_{0}^{t^{\gamma}} f^{*}\left(t^{\gamma}\right) y^{1 / p_{1}-1} d y\right)^{q} \frac{d t}{t}\right)^{1 / q}\right] .
\end{aligned}
$$

Again changing variables and then using (1), we majorize the last sum by

$$
(2 K) 2^{1 / p^{\prime}}|\gamma|^{-1 / q}\left(\frac{B_{0}}{\frac{1}{p_{0}}-\frac{1}{p}}+\frac{B_{1}}{\frac{1}{p}-\frac{1}{p_{1}}}+p_{1} B_{1}\right)\|f\|_{p q}^{*} .
$$

The remaining cases are

Case 2. $p_{1}<\infty$ and $q=\infty$,

Case 3. $p_{1}=q_{1}=\infty$ and $q<\infty$, and

Case 4. $p_{1}=q_{1}=\infty$ and $q=\infty$.

The proof of Case 2 is straightforward. Cases 3 and 4 follow, with slight change, the proofs of Cases 1 and 2 .

It is easy to see that (11), even for $T$ linear does not imply (12) when $s<q$. Let $M=(0, \infty)$ and $m=$ Lebesgue measure. Define $T f(x)$ 
$=x^{-\alpha-1} \int_{0}^{x} f(y) d y, \alpha>0$. Then $|T f(x)| \leqq x^{-\alpha} f^{* *}(x)$, so that $(T f)^{*}(x)$ $\leqq x^{-\alpha} f^{* *}(x)$, and, hence, $\|T f\|_{p_{q}}^{*_{\prime}} \leqq\|f\|_{p q} \leqq(p /(p-1))\|f\|_{p q}^{*}$, where $0<1 / p^{\prime}=1 / p+\alpha<1$. If $f$ is non-negative and nonincreasing then $f^{*}(x)=f(x)$ and $(T f)^{*}(x)=x^{-\alpha} f^{* *}(x) \geqq x^{-\alpha} f^{*}(x)$, so that $\|T f\|_{p}^{*}$, $\geqq\|f\|_{p s}^{*}$. One can easily find an $f$ such that $\|f\|_{p s}^{*}=\infty$ and $\|f\|_{p q}^{*}<\infty$ and hence (12) is impossible for any finite $B_{\theta}$.

We see now that the classical Marcinkiewicz Theorem can be extended slightly as follows:

If $T$ is of weak type $\left(p_{i}, p_{i}^{\prime}\right), 0<p_{i}, p_{i}^{\prime} \leqq \infty$, then $T$ is of type $\left(p_{\theta}, p_{\theta}^{\prime}\right)$ if $p_{\theta} \leqq p_{\theta}^{\prime}$. If $p_{\theta}>p_{\theta}^{\prime}$, the example given shows that $T$ cannot be expected to be of type $\left(p_{\theta}, p_{\theta}^{\prime}\right)$; that is, not only does the proof fail in the "upper triangle," but so does the result.

\section{BIBLIOGRAPHY}

1. A. Calderón, Intermediate spaces and interpolation, Studia Math. 1 (1963), 31-34.

2. G. H. Hardy, J. E. Littlewood and G. Pólya, Inequalities, University Press, Cambridge, 1934.

3. S. G. Krel̆n and E. M. Semenov, On a space scale, Soviet Math. Dokl. 2 (1961), 706-710.

4. J.-L. Lions and J. Peetre, Sur une classe d'espaces d'interpolation, Inst. Hautes Etudes Sci. Publ. Math. No. 19 (1964), 5-68.

5. G. G. Lorentz, Some new functional spaces, Ann. of Math. (2) 51 (1950), 37-55.

6. R. O'Neil, Convolution operators and $L(p, q)$ spaces, Duke Math. J. 30 (1963), 129-142.

7. J. Peetre, Nouvelles propriétés d'espaces d'interpolation, C. R. Acad. Sci. Paris 256 (1963), 1424-1426.

8. E. M. Stein and G. Weiss, An extension of a theorem of Marcinkiewicz and some of its applications, J. Math. Mech. 8 (1959), 263-284.

9. A. Zygmund, Trigonometric series, Vol. II, 2nd. ed., Cambridge Univ. Press, New York, 1959.

WASHINGTON UNIVERSITY 